\title{
INTENCIÓN EMPRENDEDORA DE LOS EMPLEADOS DEL SECTOR PRIVADO DE LA CIUDAD DE MEDELLÍN Y SU ÁREA METROPOLITANA
}

\author{
Entrepreneurial intention of employees in the private sector in Medellin and its metropolitan \\ area
}

\begin{abstract}
Diana Milena Hernández-López
Estudiante de Administración Tecnológica - Instituto Tecnológico Metropolitano, MedellínColombia, dianahernandez87511@correo.itm.edu.co

John Fredy Moncada-Toro iD

Estudiante de Administración Tecnológica - Instituto Tecnológico Metropolitano, Medellín-

Colombia, johnmoncada62875@correo.itm.edu.co
\end{abstract}

\author{
Laura Cristina Henao-Colorado iD \\ MSc en Administración, Instituto Tecnológico Metropolitano, Medellín- Colombia, \\ laurahenao@itm.edu.co
}

\section{Cómo citar / How to cite}

Hernández-López, D., Moncada-Toro, J. F. \& Henao-Colorado, L. C. (2018). Intención emprendedora de los empleados del sector privado de la ciudad de Medellín y su área metropolitana. Revista CEA, 4(8), 13-33. https://doi.org/10.22430/24223182.1045

Recibido: 12 de febrero de 2018

Aceptado: 7 de mayo de 2018

\section{Resumen}

La investigación relacionada a continuación, tuvo como objetivo identificar si existe intención emprendedora por parte de los empleados del sector privado de la ciudad de Medellín y su área metropolitana, desde un conjunto de variables cognitivas y sociopersonales. Para ello, se llevó a cabo una encuesta, la cual logró una muestra de 120 personas, y posteriormente se procedió a identificar qué variables influyen en la intención emprendedora de la población estudiada y analizar sus características según los factores identificados. Se concluye que existe intención emprendedora por parte de las personas que se encuentran empleadas actualmente en alguna empresa del sector privado.

Palabras clave: intención emprendedora, emprendimiento, teoría del comportamiento planificado.

\section{Abstract}

This study aims to establish if employees in the private sector in Medellín and its metropolitan area have entrepreneurial intention based on a set of cognitive and sociopersonal variables. A survey included 120 participants to identify the variables that influence the entrepreneurial intention of the population under study and analyze their characteristics according several factors. The results confirm that individuals in 
the private sector are significantly prone to start their own businesses.

Keywords: entrepreneurial intention, entrepreneurship, theory of planned behavior.

\section{INTRODUCCIÓN}

En el contexto de hoy, es inminente la importancia que tiene el fomento de la iniciativa emprendedora para generar empleo, y con ello riqueza y bienestar social para el país; esto, porque el emprendimiento se ha convertido en una alternativa que contribuye significativamente a incrementar la riqueza de los países, con la generación de empleos y por tanto con el bienestar en la población. Para Colombia, esto no es diferente, y en iniciativas de emprendimiento es un país que se destaca frente al mundo con un tercer lugar, de acuerdo con Global Entrepreneurship Monitor, cuyo reporte fue liderado por el Babson Commege y el London Business School (Amorós, 2011).

Dado lo anterior, la cultura del emprendimiento implica que se actúe hacia la creación de empresa aprovechando las oportunidades que se puedan dar, de manera que, como resultado, se obtengan beneficios para los emprendedores desde la creación de valor, beneficios para la sociedad con posibles oportunidades de empleo y por tanto para el país.

Además de establecer la importancia de emprender, y que Colombia es un país emprendedor, también se debe mirar un escenario no tan positivo y que es una realidad para las empresas de hoy, y que se relaciona con las altas tasas de rotación de personal, que enciende las alarmas para la gerencia de hoy. Dicha rotación, además de relacionarse con la inconformidad de algunas personas con aspectos de la empresa o cargo ejercido, puede estar relacionada con que muchas personas se van a emprender en sus propios negocios, resultando en altos costos para la empresa (Ramírez, 2016). Es por ello que es de vital importancia para mantener capital humano importante en las compañías, saber si las personas se están yendo por emprender, y así crear estrategias para impulsar dicha intención emprendedora al interior de la empresa y no por fuera, de manera que no sea muy costoso perder personal valioso e incluso potencializar la empresa frente a la competencia, gracias a las habilidades emprendedoras de sus trabajadores.

Este trabajo proporciona una contribución interesante a la literatura del emprendimiento, y tiene que ver con el objeto de estudio, dado que existen a nivel nacional y latinoamericano sobre intención emprendedora, investigaciones que solo apuntan a muestras conformadas por jóvenes universitarios o de secundaria (Lanero, Vázquez \& Muñoz, 2015; Barreto, Zúñiga \& Ruiz, 2016; Torres, Valencia, Bermúdez, Díez, Urrego y Maussa, 2016), no se encontraron estudios sobre la intención emprendedora en una población conformada por personas que ya se encuentran laborando para alguna empresa privada. Así, el objetivo de la presente investigación es explorar y describir, dentro del recurso humano de las empresas privadas de la ciudad de Medellín y su área metropolitana, las características de esta población en cuanto al emprendimiento y si existe o ha existido alguna intención emprendedora. En la primera parte, se muestra todo el sustento teórico de la investigación; seguido, se muestra la metodología empleada, que implicó una investigación exploratoria y descriptiva, usando como herramienta una encuesta virtual; en la tercera parte, se muestran los resultados y la discusión y por último las conclusiones. 


\section{MARCO TEÓRICO}

El emprendimiento, además de ayudar a crear empleo y al crecimiento económico del país, mejora la calidad de vida de las personas. Las empresas desarrollan sus actividades en entornos demasiados competitivos y se exponen a la globalización, a los nuevos y constantes cambios del mercado, a la innovación, a todo lo referente al servicio al cliente, etc., implicando que el emprendimiento se convierta en una solución para activar la economía. Así, existen estudios que han demostrado lo importante que es el emprendimiento para que se potencie el desarrollo económico y social del mundo entero (Rueda, Sánchez, Herrero, Blanco, \& Fernández, 2013; Ortiz-Delgadillo, EsquivelAguilar \& Hernández-Castorena, 2016).

Dicho emprendimiento, en los últimos años, ha sido tendencia en investigaciones que han generado mucho valor tanto académico como científico, y han aportado a que los conceptos de emprendimiento evolucionen. Una definición importante, fue la propuesta por Shumpeter (1911), donde definió a un emprendedor, como una persona innovadora, la cual rompe con las rutinas (Rueda et al., 2013, p. 3); y actualmente, se puede decir que un emprendedor es una persona que está lista para tomar decisiones o iniciar algo. «EI emprendimiento es una de las actividades humanas más complejas, pues en ella convergen factores socio-personales, cognitivos, económicos, políticos y culturales, que, al combinarse, pueden resultar como predictores eficaces de iniciativas de emprendimiento o de la intención emprendedora en los individuos» (Durán \& Arias, 2015, p. 322). En Colombia, la creación de empresas es considerada como un mecanismo válido para alcanzar estabilidad y seguridad laboral, y aunque conlleva una serie de riesgos a nivel económico, se percibe como una opción segura para alcanzar un ingreso estable (Rodríguez \& Prieto, 2009, p. 87).

Zhang et al. (como se citó en Diez, 2016, p. 44) propone que los emprendedores nacen y no se forman con base en la educación, sino que es una predisposición individual genética hacia él. Sánchez, Benavides \& Luna-Arocas (citado por Díez, 2016, p. 44) manifiestan que el emprendedor es el individuo que asume el riesgo y la responsabilidad de acometer y llevar a la práctica un proyecto o una idea innovadora, recopilando los recursos y capacidades necesarios con la esperanza de conseguir beneficios económicos. Por lo tanto, y para efectos de esta investigación, se considera al emprendedor como un "creador» apto para generar ideas potenciales y llevarlas a la práctica.

El emprendimiento está ligado a las intenciones, y dicha intención puede definirse como la concentración que tiene una persona para cumplir algún objetivo, como crear una empresa o algún negocio, teniendo cierto dominio e influencia en la acción, para lograr el objetivo propuesto (Valencia, Montoya \& Montoya, 2015; Montoya, Valencia \& Montoya, 2016). Para Ajzen \& Fishbein (2005), las intenciones provienen de las creencias del comportamiento humano, las cuales no necesariamente son veraces, ya que pueden ser inexactas, sesgadas o irracionales; pero, luego de que estas creencias se forman, proporciona la base cognitiva a partir de la cual se supone que las actitudes, las normas sociales percibidas y las percepciones de control -y, en última instancia, intenciones- se siguen de manera razonable y consistente.

Dichas creencias las definen como la actitud, la norma subjetiva y la percepción de control, y estos conceptos forman la Teoría del Comportamiento Planificado.

De acuerdo con Mendes \& Santos (2014), esta teoría plantea que una intención, determinada 
por los factores actitud, norma subjetiva y percepción de control, es antepuesta a cualquier tipo de comportamiento planificado. Además, esta teoría se basa en que un individuo puede tomar decisiones de una forma racional, utilizando la información que esté disponible y pensando en la implicación de sus acciones antes de resolver comportarse de determinada forma.

La definición de dichos factores, se relacionan a continuación:

- Actitud: acción de crear un negocio o empresa.

- Norma subjetiva: presión social que pueda tener un individuo, para la creación de una empresa.

- Control del comportamiento percibido: apreciación de cuán fácil o difícil puede ser la realización de un comportamiento para una persona.

Así, el primer factor, la actitud hacia el comportamiento, se refiere al grado en que una persona tiene una valoración favorable o desfavorable de la conducta en cuestión. El segundo, es un factor social que se refiere a la percepción de la presión social para realizar o no realizar la conducta; y el tercero, es el que está relacionado con la facilidad o dificultad percibida de la realización de la conducta (Marulanda, Montoya \& Vélez, 2014, p. 214). Estos factores juntos son muy importantes para que se genere una intención emprendedora, y asíllegar a la creación de una empresa o un negocio.

La Teoría del Comportamiento Planificado, por tanto, se centra en la intención del individuo para realizar una conducta determinada, reflejando dichas intenciones factores motivacionales que influyen en un comportamiento y son indicaciones del nivel de esfuerzo que las personas están dispuestas ejercer con el fin de realizar la conducta; así, una intención de comportamiento se traducirá en ejecución del mismo solo si dicho comportamiento está bajo control de la voluntad, pero sin dejarse de lado el hecho de que la ejecución depende también de otros factores, como la disponibilidad de oportunidades y recursos (tiempo, dinero, habilidades, cooperación de los demás) (Marulanda et al., p. 214).

Dado lo anterior, Ajzen \& Fishbein (2005), proponen como regla general que, entre más favorable la actitud y la norma subjetiva con respecto a un comportamiento, y cuanto mayor es el control del comportamiento percibido, más fuerte debe ser la intención del individuo para ejecutar la conducta en cuestión:

es importante darse cuenta de que las creencias conductuales, normativas y de control que las personas tienen sobre el desempeño de un determinado comportamiento, están influenciadas por una gran variedad de factores culturales, personales $y$ situacionales. Por lo tanto, podemos encontrar diferencias en las creencias entre hombres y mujeres, jóvenes y viejos, blancos y negros, educados y sin educación, ricos y pobres, dominantes y sumisos, tímidos y extrovertidos, y entre individuos que tienen un individualismo y aquellos que tienen una orientación hacia el colectivismo. Además, pueden verse afectados por el entorno físico, el entorno social, la exposición a la información, así como por disposiciones tan amplias como los valores y los prejuicios (p. 194).

Adicional a la Teoría del Comportamiento Planificado, para la década de los 80 , también se iniciaron otros modelos sobre la intención emprendedora. Uno de estos modelos fue el del evento emprendedor de Shapero y Sokol, el 
cual propone "que la decisión de iniciar una actividad emprendedora, requiere de una creencia preexistente de que dicha actividad es anhelada y realizable, unida con alguna propensión personal a actuar sobre las oportunidades y algún tipo de factor predominante» (Díez, 2016, p. 44). Como lo indica Marulanda et al. (2014), Shapero explica que la decisión de crear empresa es el producto de dos percepciones: conveniencia o deseabilidad y factibilidad o viabilidad.

Las percepciones de conveniencia tienen que ver con los valores, y son el resultado de la posición de la persona en la matriz de cultura, estructura socioeconómica, familia, educación, pares y personas influyentes [...] son más propensas a la creación de empresas aquellas culturas que valoran el ser negociante, el emprendimiento y los comportamientos asociados a estas actividades, como la independencia, la innovación y la toma de riesgos ( $p$. 216); [...] y la percepción de factibilidad, es considerada como una amalgama de conocimientos, recursos, garantías y experiencia obtenida en un negocio particular o en la educación formal. Muchos emprendedores crean empresas en sectores en los cuales han laborado; asimismo, cuando se trabaja en empresa pequeña se tiene un conocimiento más integral del negocio $y$, por tanto, hay mayor propensión a crear el propio. También mejora la percepción de factibilidad el haber tenido contacto con clientes y un acercamiento al mercado (p. 217).

Apoyando lo propuesto por Shapero y lo relacionado a la factibilidad, Villafuerte y Leiva (2015, p. 41), mostraron que para un emprendedor haber trabajado en una multinacional, fue de gran utilidad porque les permitió desarrollar habilidades vitales para su emprendimiento, ya que las funciones realizadas es dichas empresas, estuvieron muy ligadas con las funciones que se realizan en un emprendimiento, e les permitió, conocer y saber cómo manejar a quienes serían sus clientes; siendo, por tanto, esta experiencia previa, esencial para impulsar a los emprendedores a crear su propio negocio.

Otro modelo fue el propuesto por Krueger y Brazeal, denominado Modelo del Potencial Emprendedor, y el cual se enfoca en «el estudio de la percepción del deseo (normas sociales) y de la viabilidad (autosuficiencia) como inicio de la intención de la creación de un negocio» (Díez, 2016, p. 45). El aporte de Krueger y Brazeal es muy importante, dado que se ha probado que la variable autoeficacia emprendedora resulta tener efecto en la intención emprendedora, porque los individuos que se consideran capaces para el emprendimiento, también tienen una actitud favorable hacia el mismo, comenzando por sentirse capaces (Durán \& Arias, 2015, p. 332). Igualmente, las variables sociopersonales, como tener experiencia laboral y estar trabajando actualmente, implican una alta probabilidad de que el individuo considere que puede replicar una idea de negocio, pues se siente con las capacidades adquiridas en sus trabajos anteriores y por ende es probable que emprenda (Durán \& Arias, 2015, p. 333), lo cual se alinea también con lo sugerido por Villafuerte y Leiva (2015).

Sobre intención emprendedora, se tienen algunos estudios en el sector educativo a nivel mundial y a nivel país. En Andalucía, España, se llevó a cabo una caracterización de la población emprendedora, donde se destaca que los emprendedores llevan a cabo tareas de gestión en sus negocios, en su mayoría son hombres con estudios universitarios, y sus motivaciones para emprender van ligadas a una oportunidad 
de mercado detectada y valorada como rentable, otros por la necesidad económica y la preferencia por trabajar por cuenta propia (Ruiz, Sanz, \& Fuentes, 2015).

Otro estudio, para precisar los factores que tienen influencia en la intención emprendedora de los estudiantes de la Universidad de Alcalá, Madrid, arrojó que dichos factores son los personales, como la edad, el género (en este caso masculino) y los familiares; es decir, los principales resultados mostraron que las características personales como ser mayor edad, pertenecer al género masculino y la existencia de algún familiar con empresa, explicaron la intención emprendedora de la población estudiada. Igualmente, la mayor valoración personal resultó ser también un factor significativo. Por el contrario, un mayor tiempo de estudios en la carrera y la percepción de la figura del empresario por parte de la muestra no resultaron siendo factores influyentes (Moreno, 2013). También, se tienen estudios que sugieren el impacto de la educación empresarial como un factor crucial que permite a los jóvenes comprender y fomentar una actitud emprendedora (Mejía, Arias y Echeverri, 2017).

Por otro lado, en México, se ha investigado sobre la intención emprendedora analizada desde el género, donde se evidencia que no hay diferencias significativas entre el desempeño de nuevos negocios comenzados por mujeres y los nuevos comenzados por hombres, pero sí en los factores iniciales con los que comienzan como el monto de capital. Adicional se evidenció diferencias entre los niveles de ingresos obtenidos y se rechazaron las hipótesis relacionadas con diferencias en estudios y edad (Elizundia, 2015).

A nivel país, en Colombia, en la Universidad del Externado, se investigó sobre liderazgo, emprendimiento innovador y la relación entre ambos. Los resultados de dicho estudio evidenciaron que la habilidad que tienen los líderes emprendedores, interviene efectivamente a la hora de la explotación de oportunidades de negocios y reconocimientos, también se concluyó, que permiten que los miembros de las organizaciones sean guiados hacia una excelente gestión del conocimiento. Finalmente, se evidenció una dicotomía entre el líder y el emprendedor concluyéndose que el liderazgo es una característica intrínseca del emprendimiento; sin embargo, el liderazgo no es por regla utilizado para emprender (Acosta, 2012).

Otra investigación a nivel país, consistió en un comparativo entre Colombia y Francia, para validar la influencia que podían tener las creencias en el comportamiento del emprendedor, y concluyó que las normas sociales y la necesidad de aceptación influyen a la hora de crear una empresa, favoreciendo así la conducta de emprender (Mora, 2011, p. 74). Como los estudios anteriores, existen varios sobre intención emprendedora en Colombia, e igualmente a nivel latinoamericano, pero abarcando a la población estudiantil (Mora, 2011; Osorio \& Londoño, 2015; Espino \& Vásquez, 2015; Soria, Zúniga \& Ruiz, 2016), no incluyendo población que se encuentre actualmente trabajando para alguna empresa del sector privado.

Con los conceptos y estudios relacionados anteriormente, se hace énfasis en la importancia de investigar la intención emprendedora, en estudiantes universitarios, dado que se les considera potenciales emprendedores en comparación con otras muestras de estudio (Durán \& Arias, 2015, p. 323). Adicionalmente, ha cobrado relevancia la investigación sobre intención emprendedora en comunidades académicas debido al potencial de desarrollo tecnológico e innovación que presentan las Spin-Off (Cadavid, Díez-Echavarría \& Valencia, 2017), lo cual ha buscado potencializarse en Colombia 
desde los recursos asignados para la ciencia, tecnología e innovación (Becerra-Arévalo, 2015).

Adicionalmente, se considera importante para la gestión empresarial, explorar en muestras conformadas por personas que se encuentran empleadas en el sector privado, si existe intención emprendedora, qué factores se involucran en dicha intención, y también es un aporte a la escasa investigación, dado que se evidenció que no hay estudios sobre la intención emprendedora por parte de personas que trabajan en el sector privado. Para efectos de esta investigación, se toma como referente el modelo propuesto por Ajzen $\&$ Fishbein (2005) y algunos factores tenidos en cuenta por Shapero y Sokol y Krueger y Brazeal.

\section{METOdOLOGÍA}

La investigación se realizó en dos fases, exploratoria y descriptiva. En la primera fase, se elaboró el marco teórico y estado del arte necesario para la comprensión del problema de investigación y el planteamiento del objetivo.

En la segunda fase se utilizó un instrumento validado con una prueba de validez y de fiabilidad de los datos, aplicado a una muestra de 120 individuos, durante el mes de septiembre y octubre de 2017. La muestra fue seleccionada por juicio de los investigadores. En esta etapa se llevó a cabo una técnica de estadística descriptiva para caracterizar las muestras e identificar las señales de relación entre las variables demográficas y los factores (por medio de Anovas de un factor), e indicios de intención emprendedora por parte de la población aquí estudiada. Con ambas partes se pretendió ampliar el conocimiento sobre el objeto de análisis, y lograr compararlo con la literatura expuesta en el marco teórico.

Para el análisis estadístico, se usó el software SPSS. La Tabla 1, recoge la ficha técnica del estudio.

Dado que la Teoría del Comportamiento Planificado constituye una base sólida en la investigación en emprendimiento (Marulanda et al., 2014), y las variables sociopersonales propuestas por Shapero y Brazeal, para esta investigación, el instrumento aplicado se basó en variables correspondientes a los factores planteados en dichas teorías, los cuales son INT= Intención $\mathrm{ACT}=$ Actitud $\mathrm{CP}=$ Control Percibido NS= Norma subjetiva - Opinión de los demás NSC=Norma Subjetiva-Competencias, empleándose una encuesta con una escala de Likert de cinco posiciones, donde 1 indica «muy bajo», 2 «bajo», 3 «ni muy bajo ni muy alto», 4 «alto», 5 «muy alto», y otra escala donde 1 indica "muy en desacuerdo», 2 "desacuerdo», 3 «neutral», 4 «de acuerdo» y 5 «muy de acuerdo». En la Anexo (Tabla 7), se muestran las variables utilizadas para medir cada factor, adaptadas al contexto estudiado, de manera que se garantizara el entendimiento del instrumento por parte de los individuos encuestados.

Tabla 1. Ficha técnica del estudio desarrollado

Table 1. Technical data of the study

\begin{tabular}{c|c}
\hline Unidad muestral & Empleados del sector privado \\
\hline Ámbito de estudio & Medellín y área metropolitana \\
\hline Método de recogida de información & Por juicio, población profesional, empleada \\
\hline Procedimiento de muestreo & $\mathrm{n}=120$ \\
\hline Tamaño muestral & Septiembre y octubre de 2017 \\
\hline Fecha del trabajo de campo &
\end{tabular}

Fuente: elaboración propia. 
Al considerar los resultados es importante hacer claridad que, por las características del método empleado, procedimiento y muestra de la investigación, los datos aquí mostrados deben ser tomados como resultados exploratorios y no como concluyentes.

\section{RESULTADOS Y DISCUSIÓN}

Fiabilidad y validez de las escalas de medida Para verificar la fiabilidad y validez del instrumento de medida se procedió a la realización del tratamiento de los datos utilizando el software SPSS. Para asegurar la validez, se procedió con la medida de adecuación muestral de Kaiser-Meyer-Olkin y la prueba de esferidad Barltlett, de manera que se garantice que, por cada variable, la comunalidad sea mayor a 0.4 , la medida de adecuación KMO mayor a 0.5 y la prueba de Barltlett en su grado de significancia debe sea menor a 0.05 (Aldás, S. f., p. 4). En la Tabla 2., se muestra que todas las escalas de medida, cumplieron con el criterio:

Tabla 2. Validez de la escala de medida

Table 2. Validity of the measurement scale

\begin{tabular}{|c|c|c|c|c|c|c|c|}
\hline Factor & $\begin{array}{c}\text { Medida de } \\
\text { adecuación } \\
\text { muestral } \\
\text { de Kaiser- } \\
\text { Meyer- } \\
\text { Olkin }\end{array}$ & $\begin{array}{c}\text { Bartlett } \\
\text { (grado de } \\
\text { significancia) }\end{array}$ & Comunalidad & Factor & $\begin{array}{c}\text { Medida de } \\
\text { adecuación } \\
\text { muestral de } \\
\text { Kaiser- } \\
\text { Meyer- } \\
\text { Olkin } \\
\end{array}$ & $\begin{array}{c}\text { Bartlett } \\
\text { (grado de } \\
\text { significancia) }\end{array}$ & Comunalidad \\
\hline INT1 & \multirow{5}{*}{0,84} & \multirow{5}{*}{0,00} & 0,84 & CP2.1 & \multirow{8}{*}{0,80} & \multirow{8}{*}{0,00} & 0,84 \\
\hline INT2 & & & 0,84 & CP2.2 & & & 0,83 \\
\hline INT3 & & & 0,82 & CP2.3 & & & 0,54 \\
\hline INT4 & & & 0,66 & CP2.4 & & & 0,41 \\
\hline INT5 & & & 0,83 & CP2.5 & & & 0,64 \\
\hline ACT1 & \multirow{8}{*}{0,90} & \multirow{8}{*}{0,00} & 0,83 & CP2.6 & & & 0,78 \\
\hline ACT2 & & & 0,84 & CP2.7 & & & 0,75 \\
\hline ACT3 & & & 0,80 & CP2.8 & & & 0,70 \\
\hline $\mathrm{ACT} 4$ & & & 0,89 & NS1 & \multirow{3}{*}{0,68} & \multirow{3}{*}{0,00} & 0,70 \\
\hline ACT5 & & & 0,98 & NS2 & & & 0,87 \\
\hline ACT6 & & & 0,71 & NS3 & & & 0,80 \\
\hline ACT7 & & & 0,81 & NSC1 & \multirow{11}{*}{0,94} & \multirow{11}{*}{0,00} & 0,62 \\
\hline ACT8 & & & 0,61 & NSC2 & & & 0,78 \\
\hline CP1 & \multirow{5}{*}{0,87} & \multirow{5}{*}{0,00} & 0,82 & NSC3 & & & 0,67 \\
\hline CP2 & & & 0,63 & NSC4 & & & 0,72 \\
\hline CP3 & & & 0,71 & NSC5 & & & 0,72 \\
\hline $\mathrm{CP} 4$ & & & 0,77 & NSC6 & & & 0,79 \\
\hline CP5 & & & 0,84 & NSC7 & & & 0,60 \\
\hline MOT1 & \multirow{9}{*}{0,86} & \multirow{9}{*}{0,00} & 0,84 & NSC8 & & & 0,76 \\
\hline MOT2 & & & 0,75 & NSC9 & & & 0,69 \\
\hline MOT3 & & & 0,60 & NSC10 & & & 0,69 \\
\hline MOT4 & & & 0,73 & NSC11 & & & 0,81 \\
\hline MOT5 & & & 0,88 & \multirow{5}{*}{\multicolumn{4}{|c|}{ Fuente: elaboración propia. }} \\
\hline MOT6 & & & 0,72 & & & & \\
\hline MOT7 & & & 0,53 & & & & \\
\hline MOT8 & & & 0,76 & & & & \\
\hline MOT9 & & & 0,65 & & & & \\
\hline
\end{tabular}


Se puede apreciar que hay consistencia interna basada en el promedio de las correlaciones entre los ítems, dado que cumplen el criterio de que el Alpha sea mayor a 0,7. Como se puede observar, con la "Correlación elemento-total corregida», se deberían eliminar los ítems ACT5 Y CP2.4 dado que están muy cercanos a 0 y al eliminarlos mejoraría el Alpha. Para efectos de esta investigación, no serán eliminados.
Para garantizar la fiabilidad, y por tanto consistencia interna, basado en el promedio de las correlaciones entre los ítems, se calculó el Alpha de Cronbach para cada escala de cada factor (Tabla 4), y se verificó que fueran superiores a 0,7 (Vila, Küster, \& Aldás, 2000, p. 14).

Tabla 3. Fiabilidad de la escala de medida

Table 3. Reliability of the measurement scale

\begin{tabular}{|c|c|c|c|c|c|c|}
\hline $\begin{array}{c}\text { Alfa de } \\
\text { Cronbach }\end{array}$ & $\begin{array}{l}\text { Correlación } \\
\text { elemento- } \\
\text { total } \\
\text { corregida }\end{array}$ & $\begin{array}{c}\text { Alfa de } \\
\text { Cronbach si } \\
\text { se elimina el } \\
\text { elemento }\end{array}$ & Factor & $\begin{array}{l}\text { Alfa de } \\
\text { Cronbach }\end{array}$ & $\begin{array}{c}\text { Correlación } \\
\text { elemento- } \\
\text { total } \\
\text { corregida }\end{array}$ & $\begin{array}{c}\text { Alfa de } \\
\text { Cronbach si } \\
\text { se elimina el } \\
\text { elemento }\end{array}$ \\
\hline \multirow{5}{*}{0,936} & 0,86 & 0,92 & CP2.1 & \multirow{8}{*}{0,853} & 0,47 & 0,85 \\
\hline & 0,87 & 0,91 & CP2.2 & & 0,45 & 0,85 \\
\hline & 0,84 & 0,92 & CP2.3 & & 0,61 & 0,83 \\
\hline & 0,72 & 0,94 & CP2.4 & & 0,37 & 0,86 \\
\hline & 0,86 & 0,92 & CP2.5 & & 0,67 & 0,83 \\
\hline \multirow{8}{*}{0,909} & 0,84 & 0,89 & CP2.6 & & 0,78 & 0,81 \\
\hline & 0,87 & 0,88 & CP2.7 & & 0,74 & 0,82 \\
\hline & 0,84 & 0,89 & CP2.8 & & 0,67 & 0,83 \\
\hline & 0,89 & 0,88 & NS1 & \multirow{3}{*}{0,857} & 0,66 & 0,89 \\
\hline & 0,14 & 0,95 & NS2 & & 0,82 & 0,72 \\
\hline & 0,74 & 0,90 & NS3 & & 0,73 & 0,80 \\
\hline & 0,84 & 0,89 & NSC1 & \multirow{11}{*}{0,957} & 0,74 & 0,96 \\
\hline & 0,68 & 0,90 & NSC2 & & 0,86 & 0,95 \\
\hline \multirow{5}{*}{0,916} & 0,84 & 0,89 & NSC3 & & 0,78 & 0,95 \\
\hline & 0,70 & 0,92 & NSC4 & & 0,81 & 0,95 \\
\hline & 0,76 & 0,90 & NSC5 & & 0,81 & 0,95 \\
\hline & 0,80 & 0,90 & NSC6 & & 0,86 & 0,95 \\
\hline & 0,85 & 0,88 & NSC7 & & 0,73 & 0,96 \\
\hline \multirow{9}{*}{0,901} & 0,72 & 0,89 & NSC8 & & 0,84 & 0,95 \\
\hline & 0,70 & 0,89 & NSC9 & & 0,79 & 0,95 \\
\hline & 0,67 & 0,89 & NSC10 & & 0,79 & 0,95 \\
\hline & 0,73 & 0,89 & NSC11 & & 0,87 & 0,95 \\
\hline & 0,77 & 0,89 & & & & \\
\hline & 0,69 & 0,89 & & & & \\
\hline & 0,64 & 0,89 & & & & \\
\hline & 0,63 & 0,89 & & & & \\
\hline & 0,62 & 0,90 & & & & \\
\hline
\end{tabular}


trumento y existe asociación de ítem con ítem (Ver tabla 3.).

\section{Radares de percepción}

Intención y Actitud emprendedora: su grado de significancia menor a 0.05 . Se concluye, que la muestra es adecuada al ins-

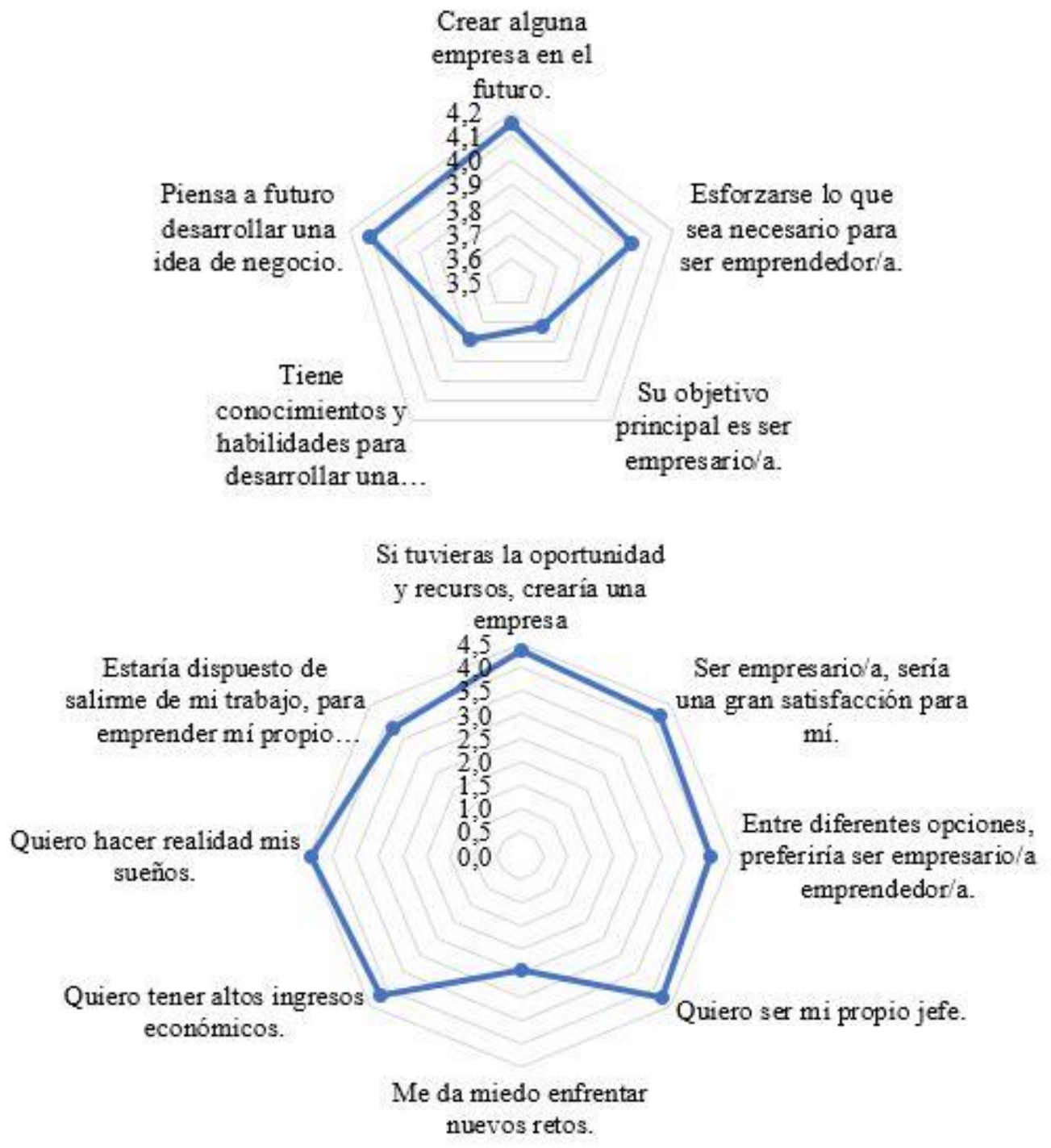

Figura 1. Radares de percepción INT y ACT

Figure 1. Radar chart of INT and ACT

Fuente: elaboración propia.

Referente a la Intención Emprendedora, dentro del personal encuestado, se ve claramente que todos coinciden en crear una empresa en el futuro. También, el deseo de emprender está muy ligado al esfuerzo que cada quien pueda aplicar a la realización de su idea. No se le entrega relevancia el hecho de no tener un conocimiento de cómo implementar y sostener una idea de negocio, la intención allí es más importante que el conocimiento o la 
experiencia que se pueda tener al ejecutar la idea de negocio o enfrentar un emprendimiento. En cuanto a la actitud, está determinada para la población encuestada por los recursos, seguido de la satisfacción que causaría en ellos poder ser sus propios jefes, pero ninguna de estas condiciones está asociada en muchos de los encuestados a tener altos ingresos económicos. Sin embargo, ven en emprender una manera de tener recursos adicionales a los que perciben en la actualidad, lo cual concuerda con lo planteado por Durán \& Arias (2015). Ver Figura 1.

Control percibido (conocimientos y habilidades) y motivación.
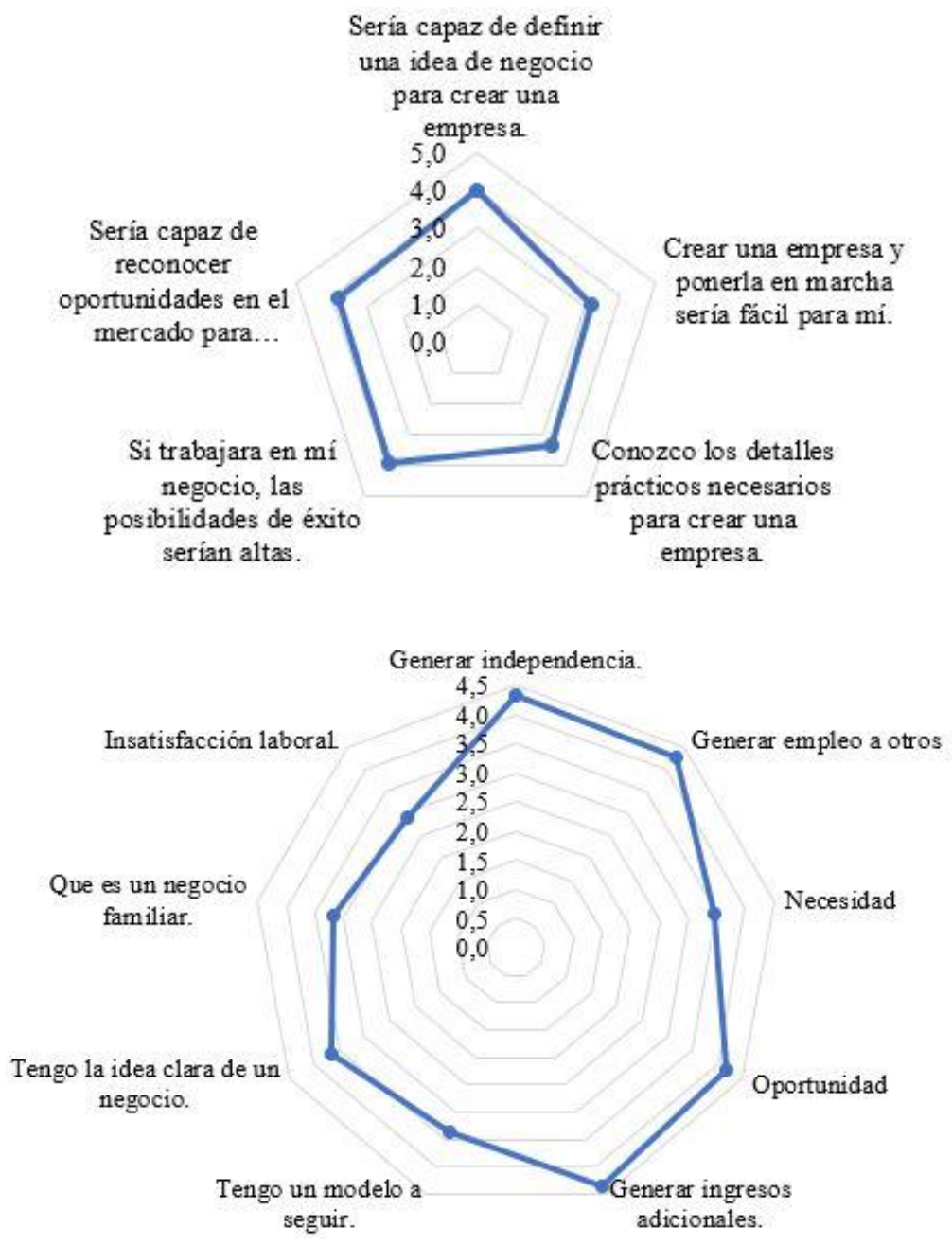

Figura 2. Radares de percepción CP1 y MOT

Figure 2. Radar chart of $\mathrm{CP} 1$ and $\mathrm{MOT}$ Fuente: elaboración propia.

Para el Control Percibido, los encuestados dicen estar en capacidad de iniciar su negocio aprovechando las oportunidades que perciben en el mercado, con una media de respuesta de 
4. Sin embargo, con una media de 3,2 en su respuesta, les es indiferente el hecho de que sea fácil o difícil emprender. Por el lado de la motivación, generar independencia es la motivación principal, seguido de poder ayudar con el desempleo actual y la insatisfacción laboral en algún aspecto en su trabajo; además, ven en emprender una manera de tener recursos adicionales a los que perciben en la actualidad. Ver Figura 2.

También, un hecho que los motiva es tener un modelo a seguir o contar con una familia que posea un negocio.

Norma subjetiva y norma subjetiva comportamiento:

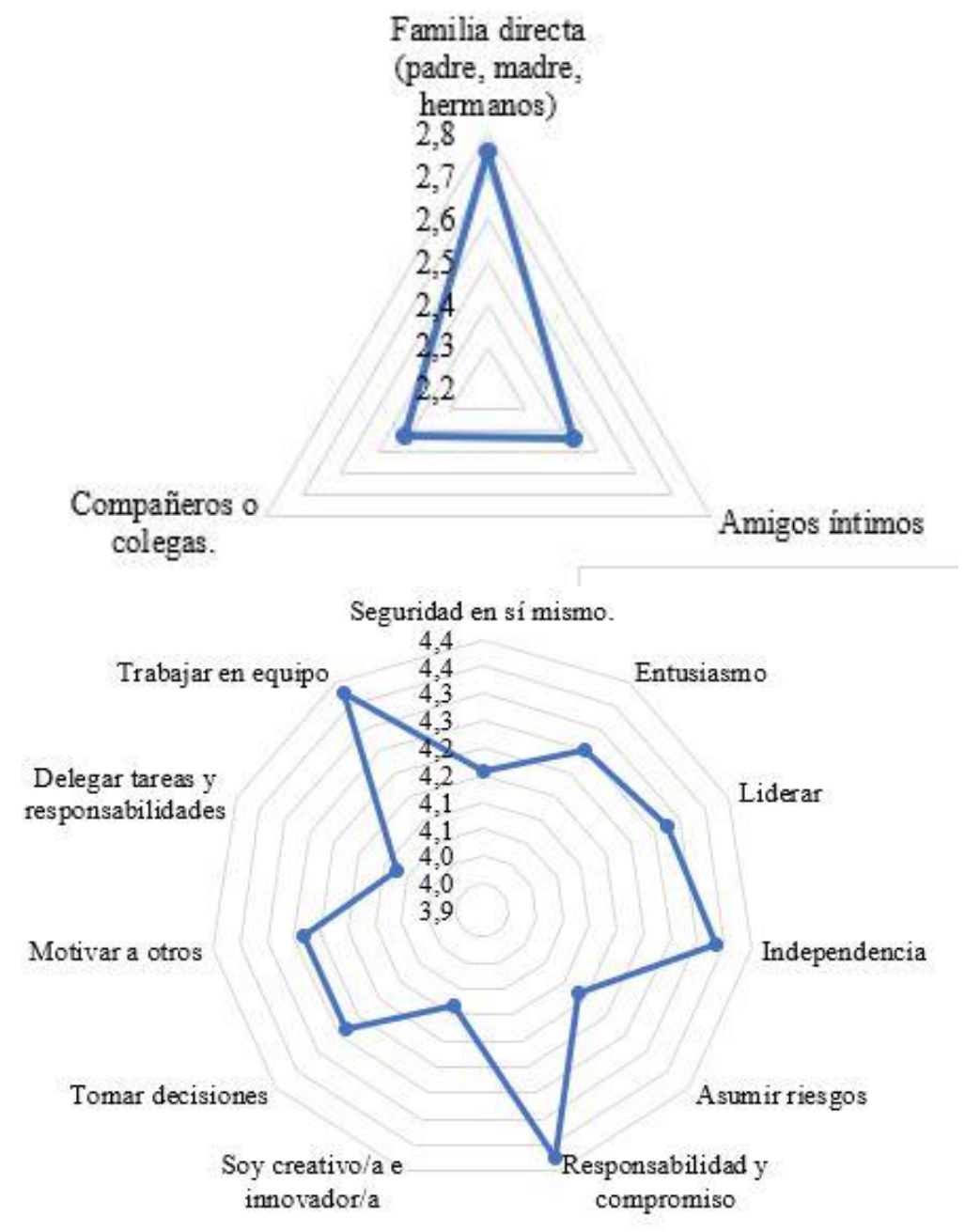

Figura 3. Radares de percepción NS y NSC

Figure 3. Radar chart of NS and NSC

Fuente: elaboración propia.

Para el caso de la Norma Subjetiva - Opinión de los demás, la media de respuesta fue 2 (en desacuerdo), no siendo ni la familia, ni los amigos y colegas, muy representativa a la hora de decidir emprender. Y por el lado de la Norma Subjetiva - Competencias, la media de 
repuesta fue 4 (de acuerdo), mostrándose que, si se crea una empresa, trabajar en equipo hace parte de la sinergia que se debe generar dentro del ambiente laboral, seguido de ser buenos líderes y tener independencia, asumiendo responsabilidades y compromisos. Adicional, tomar decisiones y asumir los riesgos están implícitos en ser emprendedores. Ver Figura 3.

\section{Control percibido (Dificultades o inconvenientes para emprender):}

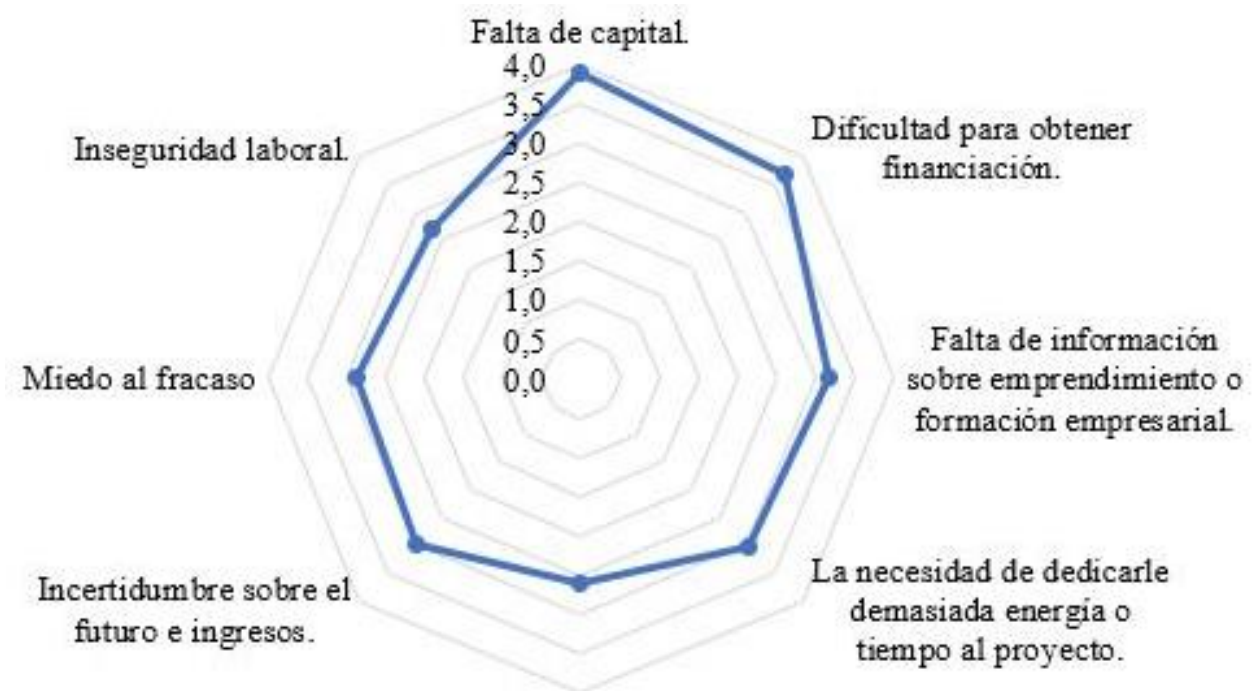

No se siente preparado.

Figura 4. Radares de percepción CP2

Figure 4. Radar chart of CP2

Fuente: elaboración propia.

Por último, en la Figura 4, se muestra lo referente al Control Percibido - dificultades / inconvenientes, donde la falta de capital reprime el deseo de emprender, y es manejado como el más alto nivel de dificultad para sacar adelante un emprendimiento, resultado que complementa lo dicho por Elizundia (2015), y que indica que los factores iniciales como el monto de capital, influyen en la intención; la incertidumbre que se genera al tener el negocio propio y las responsabilidades que acarrea, hace que la población considere que existe un alto nivel de inseguridad laboral al enfrentar un panorama como emprendedor ligado, al temor al fracaso, temor al cambio y a lo incierto que puede llegar a ser inicialmente ser dueño de su propio negocio.

\section{Análisis de medias. Anova de un factor}

Luego de analizar los factores estudiados aquí, y los cuales se tomaron como factores dependientes respecto a las variables demográficas (independientes), se procedió a hacer análisis de Anova de un factor, siguiendo un nivel de confianza del $95 \%$ y un nivel de significancia del 0,05, de manera que se pudiera señalar si hay alguna relación entre estas variables dependientes e independientes, con base en las medias.

Al contrastar los factores «Intención Emprendedora», "Actitud Emprendedora», "Control Percibido», «Control Percibido Dificultades/Inconvenientes», «Motivaciones», 
«Norma Subjetiva - Opinión de los demás» y "Norma Subjetiva - Competencias», con las variables demográficas "Género», «Edad», «Nivel de escolaridad», "Nivel de escolaridad padres», «Nivel de ingresos», «Estrato», «Estado Civil», "Círculo familiar» y «Padres dueños de un negocio", se tiene que las medias de la variable dependiente son iguales en cada grupo o categoría de la variable independiente, dando indicio de que no hay relación entre las variables, a excepción de las presentadas en las Tablas 4., 5., y 6.

Tabla 4. Círculo familiar / Motivación

Table 4. Family circle / Motivation

\begin{tabular}{l|l|r|r|r|r|c}
\hline \multicolumn{2}{c|}{} & Suma de cuadrados & gl & Media cuadrática & F & Sig. \\
\hline \multirow{3}{*}{ MOT } & Inter-grupos & 8,698 & 5 & 1,74 & 2,463 & 0,037 \\
\cline { 2 - 7 } & Intra-grupos & 80,502 & 114 & 0,706 & & \\
\cline { 2 - 7 } & Total & 89,2 & 119 & & & \\
\hline
\end{tabular}

Fuente: elaboración propia. SPSS.

Tabla 5. Nivel de escolaridad / Motivación, Actitud, Norma Subjetiva - Competencias Table 5. Educational attainment / Motivation, Attitude, Subjective norm - Skills

\begin{tabular}{|c|c|c|c|c|c|c|}
\hline & & $\begin{array}{l}\text { Suma de } \\
\text { cuadrados }\end{array}$ & gl & Media cuadrática & $\mathrm{F}$ & Sig. \\
\hline \multirow{3}{*}{ MOT } & Inter-grupos & 10,041 & 5 & 2,008 & 2,892 & 0,017 \\
\hline & Intra-grupos & 79,159 & 114 & 0,694 & & \\
\hline & Total & 89,2 & 119 & & & \\
\hline \multirow{3}{*}{ ACT } & Inter-grupos & 12,039 & 5 & 2,408 & 3,216 & 0,009 \\
\hline & Intra-grupos & 85,346 & 114 & 0,749 & & \\
\hline & Total & 97,385 & 119 & & & \\
\hline \multirow{3}{*}{ NSC } & Inter-grupos & 45,11 & 5 & 9,022 & 8,202 & 0,000 \\
\hline & Intra-grupos & 125,399 & 114 & 1,1 & & \\
\hline & Total & 170,509 & 119 & & & \\
\hline
\end{tabular}

Fuente: elaboración propia. SPSS.

Tabla 6. Edad / Norma Subjetiva - Opinión de los demás

Table 6. Age / Subjective norm - Others' opinion

\begin{tabular}{l|l|r|r|r|r|c}
\hline \multicolumn{2}{l|}{} & Suma de cuadrados & gl & Media cuadrática & F & Sig. \\
\hline \multirow{3}{*}{ NS } & Inter-grupos & 10,05 & 3 & 3,35 & 2,905 & 0,038 \\
& Intra-grupos & 133,768 & 116 & 1,153 & & \\
& Total & 143,819 & 119 & & & \\
\hline
\end{tabular}

Fuente: elaboración propia. SPSS. 
Como se puede observar, y basados en que los niveles de significancia que fueron menores a 0,05 , se muestra una relación entre el círculo familiar y la motivación para emprender, el nivel de escolaridad y la motivación, actitud y norma subjetiva - competencias, siendo este último factor dependiente, el que tiene mayor relación, dado que el estadístico de Fisher $(F)$, es mayor al resto. Igualmente, se comprueba una relación entre la edad y la norma subjetiva - Opinión de los demás. Con esto, se muestran que posiblemente existe una relación entre la motivación para emprender y el círculo familiar, el nivel de escolaridad con las competencias y la actitud para emprender, agregándose algo muy interesante, y es que la edad implica que el emprender dependa de lo que piensen los demás, lo cual va correspondido con lo obtenido por Moreno (2013), quien demostró que la edad tiene implicaciones sobre la intención emprendedora.

Con todo lo anterior, se puede decir, por tanto, que las personas buscan las oportunidades del mercado y tienen la necesidad económica y la preferencia por trabajar por cuenta propia. Resultado que va alineado con lo propuesto por Ruiz, Sanz, \& Fuentes (2015). Atendiendo esta situación, han surgido opciones donde las nuevas empresas buscan dinero para sus proyectos de inversión en el mercado de dinero a partir de los excedentes y posibilidades de inversión desde otros mercados (QuirogaJuárez \& Villalobos-Escobedo, 2015).

Adicionalmente, que las personas sienten la necesidad de aceptación tal y como lo demostró Mora (2011), y quieren ser un modelo a seguir en sus familias y contar con estas. Además, este estudio muestra que los encuestados son personas que les gusta asumir responsabilidades y compromisos y que, a pesar de su preocupación por falta de capital, incertidumbre e inseguridad laboral por la estabilidad, han considerado o existe al menos la intención de emprender en un futuro; esto, porque manifiestan sentirse capaces por los conocimientos y destrezas adquiridas en sus trabajos anteriores, tal y como lo han probado Durán \& Arias (2015), y de acuerdo con lo propuesto por Brazeal y Shapero en sus Teorías.

\section{CONCLUSIONES}

Retomando el objetivo del estudio, que consistió en explorar y describir, dentro del recurso humano de las empresas privadas de la ciudad de Medellín y su área metropolitana, las características de esta población en cuanto a emprendimiento y si existe o ha existido alguna intención emprendedora, se puede decir que desde la intención en sí (ese esfuerzo para llevar a cabo un emprendimiento), la actitud (satisfacción por ser sus propios jefes y la acción de crear un negocio o empresa), norma subjetiva (presión social) y el control percibido (cuán fácil o difícil puede ser la creación de una empresa), estas personas tienen la intención de emprender. Así, este estudio aporta a la investigación sobre emprendimiento y a la poca investigación que hay sobre la intención emprendedora en una población que a hoy se encuentra vinculada a alguna empresa del sector privado.

Por tanto, se logró mostrar en esta investigación, que entre mayor es la actitud favorable por emprender, la influencia de la norma subjetiva (lo que piensan los demás del emprendedor), y el control percibido (facilidades y dificultades), es más probable que las personas emprendan, obteniéndose resultados alineados a lo planteado en la Teoría del Comportamiento Planificado de Ajzen \& Fishben (2005). A pesar de esto, es importante hacer claridad que, por las características del método empleado, procedimiento y muestra de la investigación, los datos aquí mostrados deben ser tomados como resultados no 
concluyentes, pero que abren nuevos horizontes a la investigación y aporta al estudio de la intención emprendedora, con una población (empleados del sector privado) para la cual no se ha hecho algún estudio sobre esta temática en Colombia.

Con todo lo anterior, las empresas deben implementar estrategias que impliquen impulsar emprendimientos al interior de la compañía, o al menos, lograr explotar por medio de la motivación, a los empleados para que muestren nuevas ideas, más creatividad y ello se traduzca en buenos resultados para la empresa. De no hacerse esto, seguirá dándose altos niveles de rotación de personal y fuga de talentos que pueden ser muy valiosos y aportar de manera significativa a mejorar el desempeño de la compañía. Para futuras investigaciones, se pretende proponer un modelo de ecuaciones estructurales que permita mostrar la relación entre los factores analizados en esta investigación, y poder concluir qué impulsa a esta población a emprender. Por ahora, se sugiere que existe intención emprendedora por parte de la muestra analizada aquí, y los factores que posiblemente influyen en dicha intención.

\section{REFERENCIAS}

Acosta, J. C. (2012). Liderazgo y emprendimiento innovador en nuevas empresas de base tecnológica. un estudio de casos basado en un enfoque de gestión del conocimiento. Revista de Estudios Avanzados de Liderazgo REAL. 1(1), 5-13. Recuperado de https://www.regent.edu/acad/global/pu blications/real/vol1/num1/REAL_Vol1Nu m1_pp5-13.pdf

Ajzen, I., \& Fishbein, M. (2005). The Influence of Attitudes on Behavior. In D. Albarracín, B. T. Johnson, \& M. P. Zanna (Eds.), The handbook of attitudes (pp. 173-221). Mahwah, NJ, US: Lawrence Erlbaum Associates Publishers.

Aldás M., J. (S.F.). El análisis Factorial. Universitat de Valencia. Dpto. de Dirección de Empresas "Juan José Renau Piqueras". S.F. 16p.

Amorós, J. (2011). El proyecto Global Entrepreneurship Monitor (GEM): una aproximación desde el contexto latinoamericano. Academia. Revista Latinoamericana de Administración, 46, 115. Recuperado de http://www.redalyc.org/articulo.oa?id=7 1617238002

Barreto, K. S., Zuñiga J. S., \& Ruiz, S. (2016). Educación e Intención Emprendedora en Estudiantes Universitarios: Un Caso de Estudio. Formación Universitaria, 9(1), 2534. Recuperado de https://scielo.conicyt.cl/scielo.php?script $=$ sci_arttext\&pid=S0718-

$50062016000100004 \& \operatorname{lng}=e n \& n r m=i s o \&$ tlng=en

Becerra-Arévalo, Y. M. (2015). Sistema general de regalías: nuevos recursos para la ciencia, tecnología e innovación en Colombia. Revista CEA, 1(1), 75-91. doi: 10.22430/24223182.65

Cadavid, L., Díez-Echavarría, L. y Valencia, A. (2017). Spin-off activities at higher educational institutions: Performance implications from a modeling perspective. Journal of Developmental Entrepreneurship, 22(02), 1-21. doi: https://doi.org/10.1142/S108494671750 0133

Diez, S. (2016). La Actitud Conductual en las Intenciones Emprendedoras. Revista Empresarial, ICE-FEE-UCSG, 10(2), 42-48. 
Recuperado

de

https://dialnet.unirioja.es/descarga/artic ulo/5603317.pdf

Durán-Aponte, E., \& Arias-Gómez, D. (2015). Intención emprendedora en estudiantes universitarios: integración de factores cognitivos y socio-personales. Revista Colombiana de Ciencias Sociales, 6(2), 320-340. Recuperado de www.funlam.edu.co/revistas/index.php/ RCCS/article/download/1528/pdf_21

Echeverri, L., Valencia-Arias, Benjumea, M. y Barrera, A. (2018). Factores que inciden en la intención emprendedora del estudiantado universitario: Un análisis cualitativo. Revista Electrónica Educare, 22(2), 1-19. doi: http://dx.doi.org/10.15359/ree.22-2.10

Elizundia, M. (2015). Desempeño de nuevos negocios: perspectiva de género. Contaduría y Administración, 60(2), 468$485 . \quad$ Recuperado de http://www.sciencedirect.com/science/a rticle/pii/S0186104215300103

Espino, A. \& Vásquez, S. (2015). Intención emprendedora en estudiantes universitarios de Ingeniería Industrial Administrativa de la Universidad Católica Santa María la Antigua (USMA). Invest. pens. crit., 3(3), 13-26. Recuperado de http://eventoscorporativospanama.com/ public_html/wpcontent/uploads/2018/01/13_Espino_y_ V\%C3\%A1squez_-_IPC_3_3.pdf

Lanero, A., Vázquez, L. J., \& Muñoz, A. (2015). Un modelo social cognitivo de intenciones emprendedoras en estudiantes universitarios. Analales de Psicología, 31(1), 243-259. Recuperado de http://scielo.isciii.es/scielo.php?script=sci _arttext\&pid=S0212-

\section{6}

Marulanda, F. A., Montoya, I. A., \& Velez, J. M. (2014). Teorías motivacionales en el estudio del emprendimiento. Pensamiento \& Gestión, 36, 206-238. Recuperado de http://www.redalyc.org/articulo.oa?id=6 4631418008

Mejía, J., Arias, C. y Echeverri, L. (2017). El papel de la educación en creación de empresas en el contexto universitario a partir de los estudios registrados en Scopus. Revista CEA, 3(5), 69-87. Recuperado de http://revistas.itm.edu.co/index.php/revi sta-cea/article/view/651/649

Mendes, L., \& Santos, M. D. (2014). Factores que influyen en el uso del contenido generado por el usuario en internet. Estudios y Perspectivas en Turismo, 23, 607-625. Recuperado de http://www.redalyc.org/html/1807/1807 31336010/index.html

Montoya, I., Valencia, A. y Montoya, A. (2016). Mapeo del campo de conocimiento en intenciones emprendedoras mediante el análisis de redes sociales de conocimiento. Ingeniare. Revista Chilena de Ingeniería, 24(2), 337-350. doi: https://doi.org/10.4067/S071833052016000200015

Mora, R. (2011). Estudio de Actitudes Emprendedoras con profesionales que crearon empresa. Revista EAN, 71,70-83. Recuperado de http://www.scielo.org.co/scielo.php?pid= S0120$81602011000200006 \&$ script=sci_abstrac t\&tIng=es

Moreno, J. (2013). Análisis de los factores que influyen en la intención emprendedora de 
los estudiantes universitarios. Caracciolos, 1(1), 1-12. Recuperado de http://www3.uah.es/caracciolos/index.p $\mathrm{hp} /$ caracciolos/article/view/10/8

Ortiz-Delgadillo, G., Esquivel-Aguilar, E. O., \& Hernández-Castorena, O. (2016). El impacto de la relación con el cliente y de la capacidad de valor agregado en el servicio en el rendimiento de la Pyme Manufacturera en Aguascalientes. Revista CEA, 2(4), 47-58. doi: $10.22430 / 24223182.171$

Osorio, F. F., \& Londoño, J. C. (2015). Intención emprendedora de estudiantes de educación media: extendiendo la teoría de comportamiento planificado mediante el efecto exposición. Cuad. admon.ser.organ., 28(51), 103-131. Recuperado de http://www.scielo.org.co/pdf/cadm/v28n 51/v28n51a05.pdf

Quiroga-Juárez, C. A., \& Villalobos-Escobedo, A. (2015). Análisis del comportamiento bursátil de las principales bolsas financieras en el mundo usando el análisis multivariado (Análisis de Componentes Principales PCA) para el periodo de 2011 a 2014. Revista CEA, 1(2), 25-36. doi: $10.22430 / 24223182.122$

Ramírez, A. (2016). El costo de la alta rotación de personal para las empresas. Portafolio diciembre 18 de 2016 - 11:20 a.m. Redacción EL TIEMPO. Recuperado de http://www.portafolio.co/economia/emp leo/costos-de-la-alta-rotacion-depersonal-en-las-empresas-502333

Rodríguez, C. A., \& Prieto, F. A. (2009). La sensibilidad al emprendimiento en los estudiantes universitarios. Estudio comparativo Colombia-Francia. Rev. Innovar, 19, 73-89. Recuperado de http://www.redalyc.org/pdf/818/818190 25007.pdf

Rueda, I., Sánchez, L., Herrero, A., Blanco, B. \& Fernández-Laviada, A. (2013). ¿Existen niveles adecuados de formación y financiación que incentiven la intención emprendedora? Revista FIR, 2(3), 28-38. Recuperado de https://dialnet.unirioja.es/descarga/artic ulo/4834629.pdf

Ruiz, M., Sanz, I., \& Fuentes, M. M. (2015). Alerta emprendedora y conocimiento previo para la identificación de oportunidades emprendedoras: el papel moderador de las redes sociales. Investigaciones Europeas de Dirección y Economía de la Empresa, 21(1), 47-54. Recuperado de http://www.sciencedirect.com/science/a rticle/pii/S1135252314000409

Soria, K., Zuniga, S., \& Ruiz, S. (2016). Educación e Intención Emprendedora en Estudiantes Universitarios: Un Caso de Estudio. Formación Universitaria, 9(1), 25-34. Recuperado de http://www.scielo.cl/pdf/formuniv/v9n1/ art04.pdf

Torres, J. A., Valencia, A., Bermúdez, J., DíezEchavarría, L. F., Urrego, M. L. y Maussa, F. O. (2016). Characterization of entrepreneurial intention in university students as from Systemic Entrepreneurship Intention Model: A case study. Cuadernos de Gestión, 18(2), 1-17. doi: 10.5295/cdg.160670jt

Valencia, J. A., Montoya, I. \& Montoya, A. (2015). Factores explicativos de las intenciones emprendedoras en estudiantes universitarios. Espacios, 36(5), 7. Recuperado de http://www.revistaespacios.com/a15v36 
n05/15360507.html

Valencia, A., Peláez, E., Rúa, C. \& Awad, G. (2010). Abstencionismo: ¿por qué no votan los jóvenes universitarios? Revista Virtual Universidad Católica del Norte, 31(1), 363-387. Recuperado de: http://revistavirtual.ucn.edu.co/index.ph $\mathrm{p} /$ RevistaUCN/article/viewFile/49/107

Vila, N., Küster, I., \& Aldás, J. (2000). Desarrollo y validación de escalas de medida en
Marketing. Universitat, Facultat de Ciencies Economiques i Empresarials, $2000-55 p$.

Villafuerte, R. y Leiva, J. (2015). Cómo surge y se vincula el conocimiento relacionado con el desempeño en las Pymes: un análisis cualitativo. Revista CEA,1(2), 37$48 . \quad$ Recuperado de http://revistas.itm.edu.co/index.php/revi sta-cea/article/view/123/127 


\section{ANEXO. Escalas de medida}

Tabla 7. Escalas de medida

Table 7. Measurement scales

\begin{tabular}{|c|c|}
\hline \multicolumn{2}{|l|}{ Intención Emprendedora (INT) } \\
\hline \multicolumn{2}{|l|}{ Ha considerado o ha tenido la intención de: } \\
\hline Crear alguna empresa en el futuro & \multirow{5}{*}{$\begin{array}{l}\text { (Aragón \& Baixauli, } \\
\text { 2014) }\end{array}$} \\
\hline Esforzarse lo que sea necesario para ser emprendedor/a & \\
\hline Su objetivo principal es ser empresario/a & \\
\hline Tiene conocimientos y habilidades para desarrollar una idea de negocio. & \\
\hline Piensa a futuro desarrollar una idea de negocio & \\
\hline \multicolumn{2}{|l|}{ Actitud Emprendedora (ACT) } \\
\hline \multicolumn{2}{|c|}{$\begin{array}{l}\text { Sobre su aspecto de predisposición a emprender y consecuencias de emprender, responde las siguientes } \\
\text { preguntas: }\end{array}$} \\
\hline Si tuviera la oportunidad y los recursos, me gustaría crear una empresa & \multirow{8}{*}{$\begin{array}{l}\text { (Aragón \& Baixauli, } \\
2014\end{array}$} \\
\hline Ser empresario/a, sería una gran satisfacción para mí & \\
\hline Entre diferentes opciones, preferiría ser empresario/a emprendedor/a & \\
\hline Quiero ser mi propio jefe & \\
\hline Me da miedo enfrentar nuevos retos & \\
\hline Quiero tener altos ingresos económicos & \\
\hline Quiero hacer realidad mis sueños & \\
\hline Estaría dispuesto de salirme de mi trabajo, para emprender mí propio negocio & \\
\hline \multicolumn{2}{|l|}{ Conocimientos y Habilidades (CP) } \\
\hline \multicolumn{2}{|l|}{ Sobre conocimientos y habilidades para desarrollar una idea de negocio, usted considera que: } \\
\hline Sería capaz de definir una idea de negocio para crear una empresa & \multirow{5}{*}{$\begin{array}{l}\text { (Aragón \& Baixauli, } \\
\text { 2014) }\end{array}$} \\
\hline Crear una empresa y ponerla en marcha sería fácil para mí & \\
\hline Conozco los detalles prácticos necesarios para crear una empresa & \\
\hline Si trabajara en mí negocio, las posibilidades de éxito serían altas & \\
\hline $\begin{array}{l}\text { Sería capaz de reconocer oportunidades en el mercado para nuevos productos y/o } \\
\text { servicios }\end{array}$ & \\
\hline \multicolumn{2}{|l|}{ Motivaciones, necesidades, dificultades o inconvenientes para emprender (MOT) } \\
\hline \multicolumn{2}{|l|}{ ¿Su motivación para crear empresa es o sería? } \\
\hline Generar independencia & \multirow{5}{*}{$\begin{array}{l}\text { (Da Fonseca \& } \\
\text { Salazar, 2016) }\end{array}$} \\
\hline Generar empleo a otros & \\
\hline Necesidad & \\
\hline Oportunidad & \\
\hline Generar ingresos adicionales & \\
\hline
\end{tabular}


Tengo un modelo a seguir

Tengo la idea clara de un negocio

Que es un negocio familiar

Insatisfacción laboral

\begin{tabular}{|c|c|}
\hline \multicolumn{2}{|c|}{ Dificultades o inconvenientes para emprender (CP2) } \\
\hline Falta de capital & \multirow{9}{*}{$\begin{array}{l}\text { (Da Fonseca \& } \\
\text { Salazar, 2016) }\end{array}$} \\
\hline Dificultad para obtener financiación & \\
\hline Falta de información sobre emprendimiento o formación empresarial & \\
\hline La necesidad de dedicarle demasiada energía o tiempo al proyecto & \\
\hline No se siente preparado & \\
\hline Incertidumbre sobre el futuro e ingresos & \\
\hline Miedo al fracaso & \\
\hline No me siento preparado(a) & \\
\hline Inseguridad laboral & \\
\hline \multicolumn{2}{|l|}{ Normas Subjetivas (NS) } \\
\hline \multicolumn{2}{|c|}{ En caso de decidir emprender, en qué medida, las opiniones de los demás te afectarían. } \\
\hline Familia directa (padre, madre, hermanos) & \multirow{3}{*}{$\begin{array}{c}\text { (Aragón \& Baixauli, } \\
\text { 2014) }\end{array}$} \\
\hline Amigos íntimos & \\
\hline Compañeros o colegas & \\
\hline \multicolumn{2}{|l|}{ Normas Subjetivas Competencias(NSC) } \\
\hline Seguridad en sí mismo & \multirow{11}{*}{$\begin{array}{c}\text { (Caro, Sánchez, \& } \\
\text { Romero, 2016) }\end{array}$} \\
\hline Entusiasmo & \\
\hline Liderar & \\
\hline Independencia & \\
\hline Asumir riesgos & \\
\hline Responsabilidad y compromiso & \\
\hline Soy creativo/a e innovador/a & \\
\hline Tomar decisiones & \\
\hline Motivar a otros & \\
\hline Delegar tareas y responsabilidades & \\
\hline Trabajar en equipo & \\
\hline
\end{tabular}

Fuente: elaboración propia.

INT= Intención, $\mathrm{ACT}=$ Actitud, $\mathrm{CP}=$ Control Percibido, NS= Norma subjetiva, NSC=Norma Subjetiva Competencias 